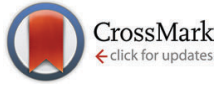

Cite this: J. Mater. Chem. C, 2017, 5, 1308

Received 9th October 2016

Accepted 20th January 2017

DOI: $10.1039 / c 6 t c 04365 h$

rsc.li/materials-c

\section{A novel angularly fused bistetracene: facile synthesis, crystal packing and single-crystal field effect transistors $\uparrow$}

\author{
Zunzhi Wang, ${ }^{a}$ Rongjin Li, ${ }^{a}$ Yulan Chen, ${ }^{a}$ Yuan-Zhi Tan, ${ }^{b}$ Zeyi Tu, ${ }^{c}$ Xuejiao J. Gao, ${ }^{d}$ \\ Huanli Dong, ${ }^{c}$ Yuanping $\mathrm{Yi}^{\mathrm{b}}{ }^{\mathrm{b}} \mathrm{Yu}$ Zhang, ${ }^{a}$ Wenping $\mathrm{Hu}$, ${ }^{\text {ac }}$ Klaus Müllen ${ }^{\mathrm{e}}$ and \\ Long Chen*a
}

\begin{abstract}
We report a facile synthesis of novel angularly fused bistetracene derivatives where two tetracene skeletons are cata-annulated at three benzene rings. Compared with previously described examples, our bistetracenes exhibit a narrower HOMO-LUMO gap but still exhibit high stability. Attempted synthesis of di-substituted bistetracene (BT-2TIPS) also led to unexpected triple (BT-3TIPS) and four-fold (BT-4TIPS) alkylsilylethynyl substitution. The photophysical, electrochemical and optical properties as well as the solid-state structure of these three bistetracene analogues are investigated. A charge carrier mobility up to $0.42 \mathrm{~cm}^{2} \mathrm{~V}^{-1} \mathrm{~s}^{-1}$ was determined based on field effect transistors.
\end{abstract}

Polycyclic aromatic hydrocarbons (PAHs) consisting of multiply fused benzene rings are among the most investigated organic semiconductors. ${ }^{1}$ It is well accepted that the electronic properties and stability of large PAHs strongly depend on the mode of ring annulation (e.g. linear or angular fusion). ${ }^{2}$ For instance, the stability of polyacenes can be greatly improved by changing their linear annulation mode to an angular one, such as in V-shaped, ${ }^{3 a}$ Z-shaped, ${ }^{3 b, c}$ and triangular analogues (Chart 1a). ${ }^{3 d}$ Recently, a concept toward two-dimensional (2D) angular PAHs was proposed by the Watkins ${ }^{4 a}$ and Briseno groups, ${ }^{4 b}$ and several newly synthesized 2D-PAHs like bisanthracene (Chart 1b) and bistetracene (Chart 1c) were used as semiconductors in FETs ${ }^{5}$ and solar cells. ${ }^{6}$ Compared to their "linear" polyacene counterparts with the same number of fused benzene rings, these new 2D-angular acenes

\footnotetext{
${ }^{a}$ Department of Chemistry, Tianjin Key Laboratory of Molecular Optoelectronic Science, Tianjin University, Tianjin 300072, China. E-mail: long.chen@tju.edu.cn

${ }^{b}$ Department of Chemistry, College of Chemistry and Chemical Engineering, Xiamen University, Xiamen 361005, China

${ }^{c}$ Institute of Chemistry, Chinese Academy of Sciences, Beijing 100190, China. E-mail: huwp@iccas.ac.cn

${ }^{d}$ Institute of High Energy Physics, Chinese Academy of Sciences, Beijing 100049, China

${ }^{e}$ Max Planck Institute for Polymer Research, Ackermannweg 10, 55128 Mainz, Germany.E-mail: muellen@mpip-mainz.mpg.de

$\dagger$ Electronic supplementary information (ESI) available. CCDC 1484734 and 1481489. For ESI and crystallographic data in CIF or other electronic format see DOI: $10.1039 / \mathrm{c} 6 \mathrm{tc} 04365 \mathrm{~h}$
}

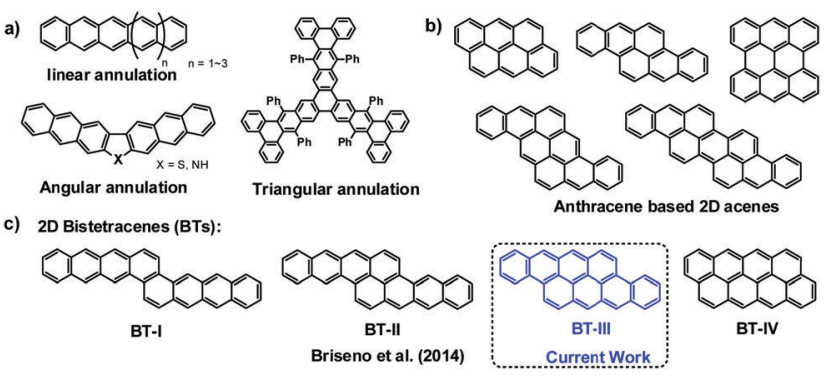

Chart 1 (a) Typical annulation modes of large PAHs; (b) typical examples of 2D-acenes composed of anthracenes; and (c) structural evolution of four bistetracenes (BTs) with different aspect ratios.

(i.e. acenes annulated at more than two rows, Chart $1 \mathrm{~b}$ and $\mathrm{c}$ ) exhibit much higher stability and maintain excellent performance like high charge carrier mobilities. ${ }^{5 a}$ Among these reported 2Dacenes, the cata-condensed analogues (e.g. bisanthracene $)^{6 a, b}$ showed higher stabilities than their peri-condensed counterparts (i.e. bisanthene). ${ }^{6 c-e}$ However, due to the inherent synthetic challenges, the number of reported 2D-angular acenes is still limited. ${ }^{4}$ We are particularly interested in 2D-angular acenes with zig-zag edges. For instance, the bistetracene (BT) should have four analogues with eight fused benzene rings which annulate at one (BT-I), two (BT-II), three (BT-III) or four (BT-IV) benzene rings (Chart 1c). These four analogues are supposed to possess different electronic structures and modes of solid-state packing due to their different aspect ratios and geometries. ${ }^{7}$ However, only the synthesis of BT-II has recently been reported. ${ }^{5 a}$ To better understand the structureproperty relationships for $2 \mathrm{D}$-acenes, ${ }^{8}$ we herein report a new bistetracene model compound (BT-III, Chart 1c), i.e. tetraceno$[2,1,12,11$-opqra $]$ tetracene, which have the same aromatic skeleton with that of Tykwinski's recent work. ${ }^{11 a}$

Compared to the known case of tetraceno[2,1,12-qra]tetracene, ${ }^{5 a}$ in which two tetracene skeletons are annulated via two neighboring benzene rings and thus create a 2D-acene analogue (Chart 1, BT-II), our new bistetracene derivatives have more annulated rings and a smaller aspect ratio, since the two tetracene skeletons are 


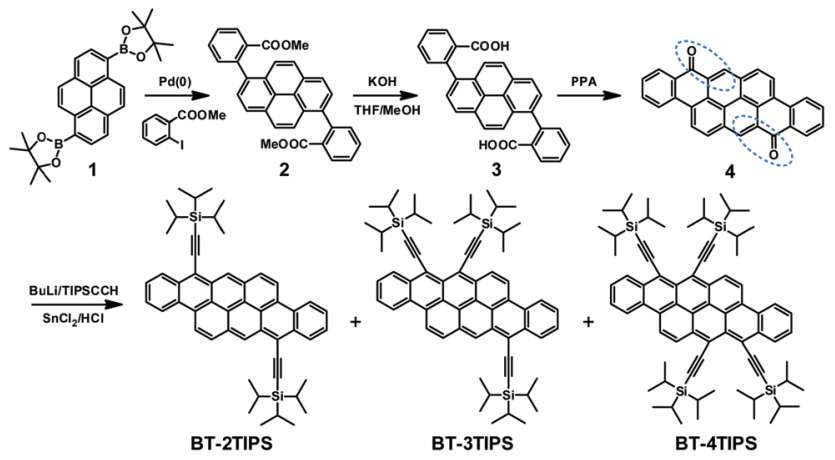

Scheme 1 Synthesis of soluble bistetracenes: BT-2TIPS, BT-3TIPS, and BT-4TIPS.

cata-condensed at three neighboring benzene rings. We used a modified procedure to synthesize the bistetracene quinone 4 as the key intermediate (Scheme 1). Suzuki coupling of 1,6-bis(4,4,5,5-tetramethyl-1,3,2-dioxaborolan-2-yl)pyrene (1) with commercially available methyl 2 -iodobenzoate produced 2 , which was subsequently hydrolyzed under basic conditions to afford the corresponding dicarboxylic acid $\mathbf{3}$ after acidification in high yield (86\%). It is noteworthy that Suzuki coupling between 1,6-dibromopyrene and methyl 2-(4,4,5,5-tetramethyl-1,3,2dioxaborolan-2-yl)benzoate also affords the dimethyl 2,2'-(pyrene1,6-diyl)dibenzoate 2 but in a much lower yield. The tetraceno[2,1,12,11-opqra]tetracene-8,16-dione (4) was obtained by intramolecular Friedel-Crafts acylation of 3 under heating with polyphosphoric acid (PPA) ${ }^{9}$ in $95 \%$ yield. We also screened other conditions like $\mathrm{SOCl}_{2} / \mathrm{AlCl}_{3}$ or Eaton's reagent; ${ }^{10}$ however, the yield was much lower with incomplete acylation intermediates and polar byproducts. ${ }^{10}$ The subsequent reaction of $\mathbf{4}$ with lithium triisopropylsilyl acetylide followed by treatment with $\mathrm{SnCl}_{2}$ afforded the target BT-2TIPS. To our surprise, MALDI-TOF MS monitoring of the reaction mixture indicated the formation of unexpected triply and fourfold triisopropylsilylethnyl substituted BTs (named BT-3TIPS and BT-4TIPS respectively). The three fractions were separated by silica gel chromatography and gel permeation chromatography (GPC). The ratio of BT-2TIPS, BT-3TIPS, and BT-4TIPs was around $13: 6: 1$, independent of the amount of lithium triisopropylsilyl acetylide (Li-CC-TIPS). The yields of BT-2TIPS, BT-3TIPS and BT-4TIPS were $26 \%, 12 \%$ and $2 \%$, respectively, probably due to the large steric hindrance between the neighboring TIPS groups. Using a large excess of Li-CC-TIPS did not improve the yield of BT-3TIPS or BT-4TIPS, which is consistent with a recent report by Tykwinski and coworkers. ${ }^{11 a}$ The plausible mechanism of two-, three-, and four-fold TIPS substitution to the bistetracene dione 4 could be ascribed to both the 1,2- and 1,4-Michael addition of the $\alpha, \beta$-unsaturated ketone moieties (highlighted in dashed ovals, Scheme 1) in 4, which was also evidenced by previous work by $\mathrm{Wu}^{11 b-d}$ and Tykwinski. $^{11 a}$

The optical properties of BT-2TIPS, BT-3TIPS, and BT-4TIPS were investigated using UV/Vis and fluorescence spectra (Fig. 1). For example, at short wavelengths, BT-2TIPS, BT-3TIPS and BT-4TIPS exhibit intense absorption bands at 354, 361 and
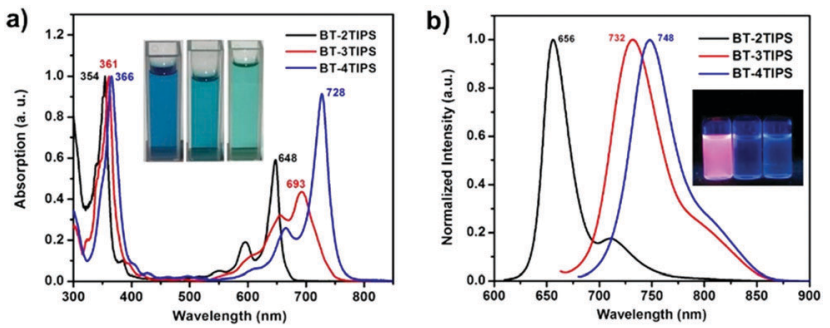

Fig. 1 (a) Normalized UV/Vis absorption spectra of BT-2TIPS, BT-3TIPS, and BT-4TIPS in THF (inset images show the photographs of their solutions under ambient light). (b) Normalized fluorescence spectra of BT-2TIPS, BT-3TIPS, and BT-4TIPS (inset images show the photographs of their solutions under $365 \mathrm{~nm}$ UV light irradiation).

$366 \mathrm{~nm}$, respectively, while in the low-energy absorption region, they all show vibrational fine absorption peaks. As obvious from Fig. 1a, BT-2TIPS display a blue color in dichloromethane (DCM) solution with an absorption peak of $\lambda_{\max }=648 \mathrm{~nm}$, while BT-3TIPS and BT-4TIPS exhibit a green color in DCM solution with $\lambda_{\max }=693$ and $728 \mathrm{~nm}$, respectively. The absorption onset for BT-2TIPS, BT-3TIPS and BT-4TIPS was determined to be 663 , 742 and $754 \mathrm{~nm}$ (Fig. S1 and Table S1, ESI $\dagger$ ), corresponding to an optical HOMO-LUMO energy gap of $1.87,1.67$ and $1.64 \mathrm{eV}$, respectively. The addition of the third TIPS-alkynyl group to BT-2TIPS results in a substantial redshift ( $45 \mathrm{~nm}$ ) of the absorption and significant lowering of the HOMO-LUMO gap (0.21 eV). In contrast, both the red shift $(35 \mathrm{~nm})$ in absorption and the band gap decrease $(0.03 \mathrm{eV})$ are much smaller upon attachment of the fourth TIPS-alkyne unit to BT-3TIPS. This could be attributed to the bending of the TIPS-alkyne branch from the mean plane of the core as evidenced by the single crystal structure discussed below. When irradiated under UV light, BT-2TIPS gave a red visible photoluminescence with a maximum at $656 \mathrm{~nm}$ and a quantum yield (QY) of 71\% (Fig. 1b inset). In sharp contrast, BT-3TIPS and BT-4TIPS are hardly emissive in the visible region but exhibit distinct near infrared emission at 732 and $748 \mathrm{~nm}$, respectively (Fig. 1b). BT-3TIPS displays a bathochromic shift of $76 \mathrm{~nm}$ in the fluorescence spectra compared to BT-2TIPS, while BT-4TIPS shows a much smaller emission redshift of $16 \mathrm{~nm}$ compared to BT-3TIPS with additional TIPSalkynyl substitution (Fig. S2, ESI $\dagger$ ). We tested the photostability of BT-2TIPS, BT-3TIPS, and BT-4TIPS by monitoring their UV/Vis absorption over time in toluene solutions $\left(1.0 \times 10^{-5} \mathrm{M}\right)$ under ambient light and air. In general, BT-2TIPS, BT-3TIPS, and BT-4TIPS are stable toward air and water (Fig. S3, ESI $\dagger$ ). In contrast, BT-2TIPS gradually degraded with a half-life time of ca. 4.5 days. However, BT-3TIPS and BT-4TIPS exhibited exceptionally high photostability, having a half-life time of 7.5 days and 54.9 days, respectively (Fig. S4, ESI $\dagger$ ). The latter is thus much more stable than the TIPS-pentacenes under similar conditions. ${ }^{12}$

To elucidate the influence of the annulation topology of the $\pi$-conjugated system and peripheral silylethnyl substitution on the molecular energy levels of these new bistetracenes, the electrochemical properties of BTs were investigated by cyclic voltammetry (CV) and differential pulse voltammetry (Fig. S5, $\mathrm{ESI} \dagger$ ). The onset potentials, together with the calculated values 
for the HOMO-LUMO energy levels of BT-2TIPS, BT-3TIPS, and BT-4TIPS, are summarized in Table S1 (ESI $\dagger$ ). For example, BT-2TIPS showed two irreversible oxidation waves and two reversible reduction waves, with the onset potential of the first oxidation at $0.25 \mathrm{eV}\left(v s . \mathrm{Fc}^{+} / \mathrm{Fc}, 4.80 \mathrm{eV}\right),{ }^{13}$ indicating a HOMO energy of $-5.05 \mathrm{eV}$. The LUMO energy was determined to be $-3.18 \mathrm{eV}$ according to the optical band gap (1.87 eV) for BT-2TIPS. As seen from Fig. S6 (ESI $\dagger$ ), DFT calculations performed at the B3LYP/6-31G(d,p) level reveal that the LUMO energies of BT-2TIPS, BT-3TIPS, and BT-4TIPS decrease when the number of TIPS substituents increases, while their HOMO energies almost remain unchanged, which results in the narrowing of their optical band gaps. These results are in good agreement with the experimental data (Table S1, ESI $\dagger$ ).

Single crystals were grown from chloroform solution by slow vapor diffusion of methanol. ${ }^{14}$ Notably, the number of substituents is critical for the packing mode in the crystalline state, although they have the same rigid skeleton fused by eight benzene rings with the same aspect ratio. The single crystal structures of BT-3TIPS and BT-4TIPS clearly confirmed three TIPS substituents at the 1, 6 and 7 positions and four TIPS substituents at the 1, 6, 7, and 12 positions (Fig. 2a) of the 2D BT-III skeleton, respectively. The molecular structures of BT-3TIPS and BT-4TIPS (Fig. S7, ESI $\dagger$ ) are both strongly distorted from planarity (Fig. S8, ESI $\dagger$ ) due to the steric hindrance of adjacent TIPS-acetylene groups. In the case of BT-3TIPS, the molecules form stacked dimers via $\pi-\pi$ stacking with a distance of $3.38 \AA$ (Fig. S7c and d, ESI $\dagger$ ), in which the $\pi$-overlap ${ }^{15}$ between face-to-face molecules amounts to more than five full benzene rings ( $c a$. 26.2 $\AA^{2}$ ), about three times more than that of 6,13-bis(TIPS-acetylene)pentacene $\left(7.7 \AA^{2}\right)^{16}$ (Fig. S9, ESI $\dagger$ ). The dimers further pack along the $a$ axis in a herringbone mode (Fig. 2c). In comparison to BT-3TIPS, the molecules of BT-4TIPS stack into a slipped one-dimensional $\pi$-stacking column with the same $\pi-\pi$ interaction distance (3.38 $\AA$ ) along the $b$ axis in the solid state (Fig. 2d), similar to previously reported BT-II ${ }^{5 a}$ and some pentacene derivatives. ${ }^{17}$ Unfortunately, we failed to obtain sufficiently large crystals of BT-2TIPS suitable for X-ray crystallographic analysis.

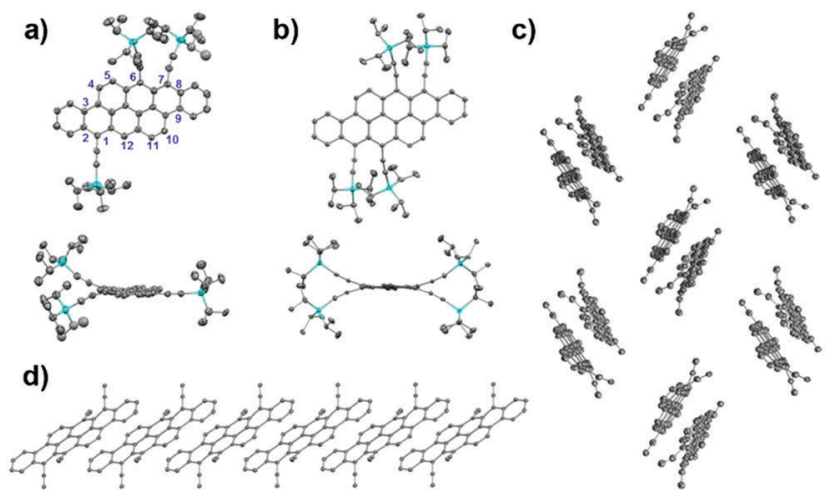

Fig. 2 Crystal structure and molecular packing of BT-3TIPS and BT-4TIPS: top and side views of (a) BT-3TIPS, and (b) BT-4TIPS (shown as ORTEP drawings; $\mathrm{H}$ atoms have been omitted for clarity). Crystal packing of (c) BT-3TIPS and (d) BT-4TIPS (triisopropylsilyl groups have been omitted for clarity).
With these solid-state structures in mind, we further evaluated their charge transport properties based on single crystal field-effect transistors with a top-contact bottom-gate configuration (for details, see the ESI $\dagger$ ). All the ribbons were formed in situ by drop casting the solution on octadecyltrichlorosilane (OTS) modified $\mathrm{SiO}_{2} / \mathrm{Si}$ substrates upon slow solvent evaporation. Gold source and drain electrodes were fabricated either by thermal vacuum deposition of gold with an organic ribbon as the shadow mask ${ }^{18}$ (for BT-2TIPS and BT-3TIPS) or by the gold layer glue technique ${ }^{19}$ (for BT-4TIPS). The OFET devices were studied under ambient conditions using a standard probe station.

The transfer and output characteristics are shown in Fig. 3. Fig. 3a-e presents the typical current-voltage characteristics for BT-2TIPS, BT-3TIPS, and BT-4TIPS, respectively. On the left axis, in black, we plot the drain current $\left(I_{\mathrm{D}}\right)$ as a function of the applied gate-to-source voltage $\left(V_{\mathrm{G}}\right)$ for a constant sourcedrain voltage $\left(V_{\mathrm{D}}=-60 \mathrm{~V}\right.$ for BT-2TIPS and BT-3TIPS or $-100 \mathrm{~V}$ for BT-4TIPS). On the right axis, in blue, the square-root value of the $I_{\mathrm{D}}$, and the slope of this curve were used for the determination of the field-effect mobility in the saturation regime. The highest mobilities of BT-2TIPS, BT-3TIPS, and BT-4TIPS are $0.42,0.25$ and $0.19 \mathrm{~cm}^{2} \mathrm{~V}^{-1} \mathrm{~s}^{-1}$, with current on/off ratios of $9.4 \times 10^{3}, 1.4 \times 10^{4}$ and $1.0 \times 10^{3}$, respectively. It is noteworthy that notable hysteresis was found for all the $I-V$ curves of the three materials, which was tentatively ascribed to the trapping of water and/or oxygen during the measurements
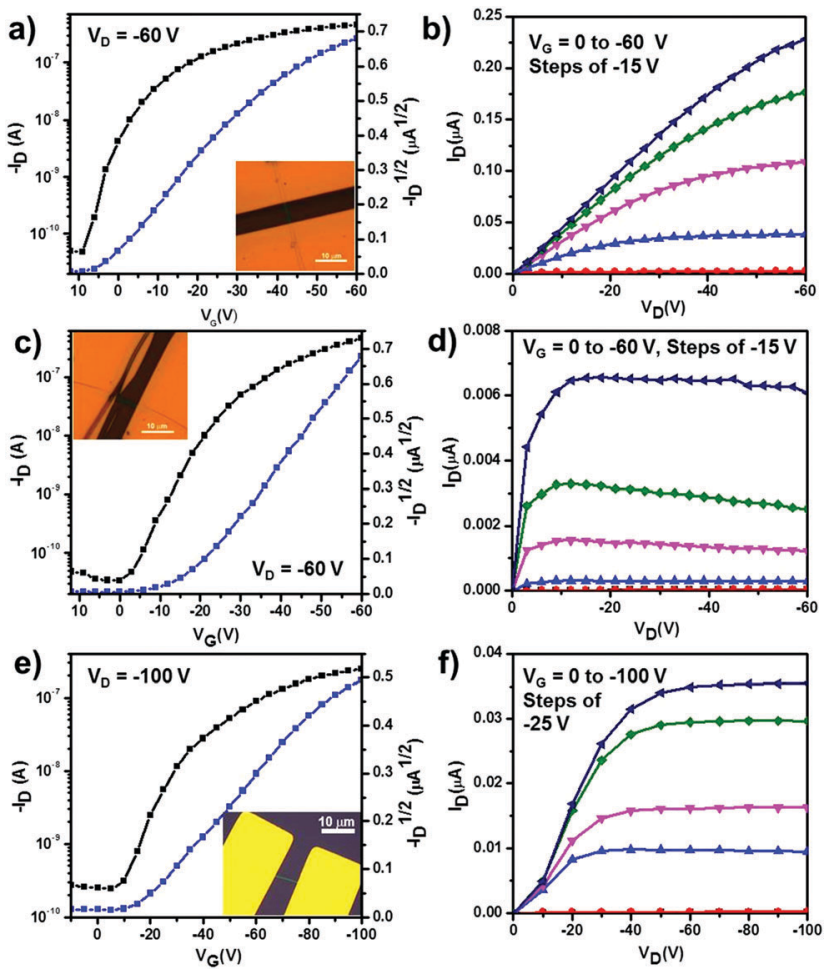

Fig. 3 Typical transfer and output characteristics of BT-TIPS based single-crystal transistors fabricated on OTS-treated $\mathrm{Si} / \mathrm{SiO}_{2}$ substrates: (a) and (b) BT-2TIPS; (c) and (d) BT-3TIPS; (e) and (f) BT-4TIPS (insets show the microscopy images of the single crystal based devices with a scale bar of $10 \mu \mathrm{m}$, for clear images see also Fig. S10a-c, ESI $)$. 
in the air (see Fig. S10f, ESI $\dagger$ for a typical hysteresis curve). ${ }^{20}$ BT-3TIPS and BT-4TIPS exhibit similar charge carrier mobilities, which are about one half of that of the BT-2TIPS. The relatively low on/off ratio might be due to the relatively large contact resistance, and is comparable to the reported 6,12-bis(trisisopropylsilylethynyl)dibenzo[def,mno]chrysene (TIPS-DBC: $2.7 \times 10^{-5} \mathrm{~cm}^{2} \mathrm{~V}^{-1} \mathrm{~s}^{-1}$, on/off ratio of $\left.10^{3}\right),{ }^{5 b}$ but with much higher mobilities. To understand the intrinsic charge carrier transport of BT- $n$ TIPS $(n=3,4)$, we investigated their electronic couplings (transfer integrals) by DFT calculations at the B3LYP/6-31G** level of theory. The determination of transfer integrals was based on the experimental crystal geometries (Fig. S10 and Table S2, ESI $\dagger)$. The transfer integrals for both holes and electrons of BT-4TIPS were found along the stacking directions ( $a$ axis) and estimated as 44 and $63 \mathrm{meV}$, respectively, while in the case of BT-3TIPS, the transfer integrals are restrained only within the $\pi-\pi$ stacked dimers with $54 \mathrm{meV}$ for holes and $41 \mathrm{meV}$ for electrons. These results indicate that the charges are easier to transport in BT-4TIPS along the 1D-stacked columns, while BT-3TIPS hold greater potential for charge hopping within the dimer stacks due to its larger transfer integrals for holes. We also investigated the local hole-vibration and electron-vibration couplings in BT- $n$ TIPS $(n=2,3,4)$. The strength of these couplings is related to the reorganization energy $(\lambda)$, where $\lambda$ is defined as the sum of intramolecular geometry relaxation energies upon changing from the neutral to the charged-state (cation/anion) geometry and vice versa. The reorganization energies were calculated from the relevant points on the potential energy surface and the results are shown in Table S3 (ESI $\dagger$ ). For all three molecules, $\lambda$ is lower for holes than for electrons. The lowest value of the reorganization energies is found in BT-2TIPS for both holes and electrons. This might account for the largest mobility of BT-2TIPS among the three molecules. For the BT-3TIPS and BT-4TIPS crystals, the theoretical hole/electron mobilities are estimated to be $0.0019 / 0.0311$ and $0.462 / 0.573 \mathrm{~cm}^{2} \mathrm{~V}^{-1} \mathrm{~s}^{-1}$ (Fig. S12, ESI $\dagger$ ), respectively. Compared with the experimental values, the hole mobility for BT-3TIPS is substantially underestimated, which is probably due to ignoring the contribution from the variance of the transfer integral induced by thermal fluctuation. As seen in Fig. S13 (ESI $\dagger$ ), the variance of the transfer integral can be very important with respect to the value of the transfer integral at the equilibrium geometry.

In summary, we have synthesized a series of novel angularly shaped 2D-bistetracene analogues based on TIPS functionalized $\mathrm{PAH}$ molecules. These bistetracene derivatives exhibit high air stability and photostability, and show distinct NIR emission and high charge carrier mobilities. Moreover, due to the large steric hindrance of TIPS groups, both the molecular planarity and packing mode of these rigid 2D molecular skeletons exhibit significant differences with additional TIPS attachments. Compared to the reported 2D-bistetracene, our title compounds indicate that simply "migrating" the aromatic sextet of cata-condensed PAH with the same number of fused benzene rings substantially affects the reactivity, ${ }^{21}$ stability and electronic properties. Further extension of the cata-condensation strategy for constructing new 2D-heteroacenes is currently underway in our laboratory.

\section{Acknowledgements}

This work was supported by the National Natural Science Foundation of China (51522303), the National Key Basic Research Program of China (2015CB856500), the Tianjin Municipal Science and Technology Commission (Grant 15JCYBJC53000) and the Thousand Youth Talents Plan. K. Müllen is grateful for the financial support from the Graphene Flagship (CNECT-ICT-604391) and Gutenberg Research College of the Johannes-Gutenberg University, Mainz.

\section{Notes and references}

1 (a) J. Wu, W. Pisula and K. Müllen, Chem. Rev., 2007, 107, 718-747; (b) M. Bendikov, F. Wudl and D. F. Perepichka, Chem. Rev., 2004, 104, 4891-4946; (c) J. E. Anthony, Chem. Rev., 2006, 106, 5028-5048; (d) J. E. Anthony, Angew. Chem., Int. Ed., 2008, 47, 452-483; (e) Z. Sun, Q. Ye, C. Chi and J. Wu, Chem. Soc. Rev., 2012, 41, 7857-7889; $(f)$ Q. Ye and C. Chi, Chem. Mater., 2014, 26, 4046-4056.

2 R. Gutzler and D. F. Perepichka, J. Am. Chem. Soc., 2013, 135, 16585-16594.

3 (a) T. V. Pho, J. D. Yuen, J. A. Kurzman, B. G. Smith, M. Miao, W. T. Walker, R. Seshadri and F. Wudl, J. Am. Chem. Soc., 2012, 134, 18185-18188; (b) B. B. Rao, J.-R. Wei and C.-H. Lin, Org. Lett., 2012, 14, 3640-3643; (c) F. IIhan, D. S. Tyson, D. J. Stasko, K. Kirschbaum and M. A. Meador, J. Am. Chem. Soc., 2006, 128, 702-703; (d) J. M. Alonso, A. D. Diaz-Alvarez, A. Criado, D. Perez, D. Peña and E. Guitian, Angew. Chem., Int. Ed., 2012, 51, 173-177.

4 (a) K. N. Winzenberg, P. Kemppinen, G. Fanchini, M. Bown, G. E. Collis, C. M. Forsynth, K. Hegedus, T. B. Singh and S. E. Watkins, Chem. Mater., 2009, 21, 5701-5703; (b) L. Zhang, Y. Cao, N. S. Colella, Y. Liang, J.-L. Brédas, K. N. Houk and A. L. Briseno, Acc. Chem. Res., 2015, 48, 500-509.

5 (a) L. Zhang, A. Fonari, Y. Liu, A.-L. M. Hoyt, H. Lee, D. Granger, S. Parkin, T. P. Russell, J. E. Anthony, J.-L. Brédas, V. Coropceanu and A. L. Briseno, J. Am. Chem. Soc., 2014, 136, 9248-9251; (b) L. Zhang, A. Fonari, Y. Zhang, G. Zhao, V. Coropceanu, W. Hu, S. Parkin, J.-L. Brédas and A. L. Briseno, Chem. - Eur. J., 2013, 19, 17907-17916; (c) L. A. Stevens, K. P. Goetz, A. Fonari, Y. Shu, R. M. Williamson, J.-L. Brédas, V. Coropceanu, O. D. Jurchescu and G. E. Collis, Chem. Mater., 2015, 27, 112-118.

6 (a) K. N. Winzenberg, P. Kemppinen, G. Fanchini, M. Bown, G. E. Collis, C. M. Forsyth, K. Hegedus, T. Birendra Singh and S. E. Watkins, Chem. Mater., 2009, 21, 5701-5703; (b) L. Zhang, B. Walker, F. Liu, N. S. Colella, S. C. B. Mannsfeld, J. J. Watkins, T.-Q. Nguyen and A. L. Briseno, J. Mater. Chem., 2012, 22, 4266-4268; (c) J. Li, K. Zhang, X. Zhang, K.-W. Huang, C. Chi and J. Wu, J. Org. Chem., 2010, 75, 856-863; (d) A. Konishi, Y. Hirao, M. Nakano, A. Shimizu, E. Botek, B. Champagne, D. Shiomi, K. Sato, T. Takui, K. Matsumoto, H. Kurata and T. Kubo, J. Am. Chem. Soc., 2010, 132, 11021-11023; (e) A. Konishi, Y. Hirao, K. Matsumoto, H. Kurata, R. Kishi, Y. Shigeta, M. Nakano, K. Tokunaga, K. Kamada and T. Kubo, J. Am. Chem. Soc., 2013, 135, 1430-1437. 
7 (a) B. T. Haire, K. W. J. Heard, M. S. Little, A. V. S. Parry, J. Raftery, P. Quayle and S. G. Yeates, Chem. - Eur. J., 2015, 21, 9970-9974; (b) D. Pérez, D. Peña and E. Guitián, Eur. J. Org. Chem., 2013, 5981-6013; (c) J. O. Oña-Ruales and Y. Ruiz-Morales, J. Phys. Chem. A, 2014, 118, 12262-12273.

8 Y. Geng, S. Sangtarash, C. Huang, H. Sadeghi, Y. Fu, W. Hong, T. Wandlowski, S. Decurtins, C. J. Lambert and S.-X. Liu, J. Am. Chem. Soc., 2015, 137, 4469-4476.

9 D. Thetford and A. P. Chorlton, Dyes Pigm., 2004, 61, 49-62.

10 (a) H. Suzuki, T. Enya and Y. Hisamatsu, Synthesis, 1997, 1273-1276; (b) Y. Zou, T. Yuan, H. Yao, D. J. Frazier, D. J Stanton, H.-J. Sue and L. Fang, Org. Lett., 2015, 17, 3146-3149.

11 (a) C. Reus, M. P. Lechner, M. Schulze, D. Lungerich, C. Diner, M. Gruber, J. M. Stryker, F. Hampel, N. Jux and R. R. Tykwinski, Chem. - Eur. J., 2016, 22, 9097-9101; (b) J. Li, C. Jiao, K.-W. Huang and J. Wu, Chem. - Eur. J., 2011, 17, 14672-14680; (c) Q. Ye, J. Chang, K.-W. Huang, G. Dai, J. Zhang, Z.-K. Chen, J. Wu and C. Chi, Org. Lett., 2012, 14, 2786-2789; (d) Q. Ye and C. Chi, Chem. Mater., 2014, 26, 4046-4056.

12 L. Abu-Sen, J. J. Morrison, A. B. Horn and S. G. Yeates, Adv. Opt. Mater., 2014, 2, 636.

13 (a) S. O. Jung, Q. Zhao, J.-W. Park, S. O. Kim, Y.-H. Kim, H.-Y. Oh, J. Kim, S.-K. Kwon and Y. Kang, Org. Electron., 2009, 10, 1066-1073; (b) Y. Zagranyarski, L. Chen, Y. Zhao, H. Wonneberger, C. Li and K. Müellen, Org. Lett., 2012, 14, 5444-5447.
14 Crystal data for BT-3TIPS, $\mathrm{C}_{126} \mathrm{H}_{152} \mathrm{Si}_{6}$ : green needle, orthorhombic, space group Pna21 (No. 33); $a=33.707(2) \AA$, $b=16.5828(12) \AA, c=19.1233(11) \AA ; \alpha=90^{\circ}, \beta=90^{\circ}, \gamma=90^{\circ}$; $V=10689.1(13) \AA^{3} ; Z=4$; final $R_{1}=0.1100(I>2 \sigma(I))$, $\mathrm{w} R_{2}=0.2958$ (all data); CCDC 1484734. Crystal data for BT-4TIPS, $\mathrm{C}_{74} \mathrm{H}_{96} \mathrm{Si}_{4}$ : green needle, triclinic, space group $P \overline{1}$; $a=7.4917(7) \AA, b=12.6697(14) \AA, c=17.4733(16) \AA ;$ $\alpha=81.367(8)^{\circ}, \beta=85.995(8)^{\circ}, \gamma=80.270(9)^{\circ} ; V=1614.5(3) \AA^{3}$; $Z=1$; final $R_{1}=0.0948(I>2 \sigma(I)), \mathrm{w} R_{2}=0.2827$ (all data); CCDC 1481489.

$15 \pi$-Overlap refers to the projected area shared between two stacked bistetracene skeletons.

16 (a) J. E. Anthony, D. L. Eaton and S. R. Parkin, Org. Lett., 2002, 4, 15-18; (b) J. E. Anthony, J. S. Brooks, D. L. Eaton and S. R. Parkin, J. Am. Chem. Soc., 2001, 123, 9482-9483.

17 J. E. Anthony, S. Subramanian, S. R. Parkin, S. K. Park and T. N. Jackson, J. Mater. Chem., 2009, 19, 7984-7989.

18 L. Jiang, J. Gao, E. Wang, H. Li, Z. Wang, W. Hu and L. Jiang, Adv. Mater., 2008, 20, 2735-2740.

19 Q. Tang, Y. Tong, H. Li, Z. Ji, L. Li, W. Hu, Y. Liu and D. Zhu, Adv. Mater., 2008, 20, 1511-1515.

20 (a) D. J. Late, B. Liu, H. S. S. Ramakrishna Matte, V. P. Dravid and C. N. R. Rao, ACS Nano, 2012, 6, 5635-5641; (b) S. H. Kim, H. Yang, S. Y. Yang, K. Hong, D. Choi, C. Yang, D. S. Chung and C. E. Park, Org. Electron., 2008, 9, 673-677.

21 Y. Cao, Y. Liang, L. Zhang, S. Osuna, A.-L. M. Hoyt, A. L. Briseno and K. N. Houk, J. Am. Chem. Soc., 2014, 136, 10743-10751. 